\title{
Anaesthesiologist-provided pre-hospital advanced airway management in children
}

\author{
M Tarpgaard", TM Hansen, L Rognås \\ From London Trauma Conference 2014 \\ London, UK. 9-12 December 2014
}

\section{Background}

Pre-hospital advanced airway management has been named one of the top-five research priorities in physician-provided pre-hospital critical care [1]. Few studies have been made on paediatric pre-hospital advanced airway management. The aim of this study was to investigate first-pass success rates and complications related to pre-hospital advanced airway management in patients younger than 16 years of age treated by prehospital critical care teams in the Central Denmark Region (1.3 million inhabitants).

\section{Method}

A prospective descriptive study based on data collected from eight anaesthesiologist-staffed pre-hospital critical care teams between February 1st 2011 and November 1 st 2012.

\section{Results}

Of a total of 25000 pre-hospital critical care missions, the pre-hospital critical care anaesthesiologists attempted endotracheal intubation in 25 children, 13 of which were less than 2 years old.

In one patient, a neonate (600g birth weight), endotracheal intubation failed. The patient was managed by uneventful bag-mask ventilation.

All other children had their tracheas successfully intubated by the pre-hospital critical care anaesthesiologists.

Over-all first pass success-rate was $75.0 \%$. In the group of patients younger than 2 years old, first pass success-rate was $53.8 \%$.

The overall rate of airway management related complications was $20 \%$ in children younger than 16 years of age and $38 \%$ in children younger than 2 years of age $(n=13)$. No deaths, cardiac arrests or severe bradycardia (heart rate $<60$ ) occurred in relation to pre-hospital advanced airway management.

\section{Discussion}

Compared to the adult population [2] the overall firstpass success rate is low. The complication rates (hypoxia, hypotension, aspiration and oesophageal intubations) in the paediatric population are higher than previously described in our pre-hospital advanced airway management patient population as a whole [2]. This illustrates that young children may represent a substantial pre-hospital airway management challenge even for experienced pre-hospital critical care anaesthesiologists.

Published: 11 September 2015

References

1. Fevang $E_{\text {, et }}$ al: The top five research priorities in physician-provided prehospital critical care: a consensus report from a European research collaboration. Scand J Trauma Resusc Emerg Med 2011, 19:57.

2. Rognas $L$, et al: Pre-hospital advanced airway management by experienced anaesthesiologists: a prospective descriptive study. Scand J Trauma Resusc Emerg Med 2013, 21:58.

doi:10.1186/1757-7241-23-S2-A22

Cite this article as: Tarpgaard et al:: Anaesthesiologist-provided prehospital advanced airway management in children. Scandinavian Journal of Trauma, Resuscitation and Emergency Medicine 2015 23(Suppl 2):A22.

* Correspondence: tarpgaard@dadlnet.dk

Department of Pre-hospital Medical Services, Central Denmark Region,

Denmark 